\title{
Decreased susceptibility to vancomycin in meticillin-resistant Staphylococcus aureus: a 5 year study in an Indian tertiary hospital
}

Currently, measures to control

Staphylococcus aureus infections are challenged by a large and continuing increase in the prevalence of meticillinresistant $S$. aureus (MRSA) worldwide (Biedenbach et al., 2004; NNISS, 2004), the spread of highly virulent communityassociated MRSA (Chapman et al., 2005; Sakoulas et al., 2004), and the emergence of vancomycin-intermediate $S$. aureus (VISA) and vancomycin-resistant $S$. aureus (VRSA) strains (Fridkin et al., 2003; Hiramatsu et al., 1997; Robert et al., 2006; Tenover, 2008). Although VISA and VRSA strains are rare among $S$. aureus clinical isolates, reports of their emergence have nonetheless alarmed the medical community. There is now also a growing concern about the emergence of $S$. aureus with increasing MICs to vancomycin. This is due to the fact that analysis of clinical and microbiological data from patients for whom vancomycin therapy failed suggests that the increasing vancomycin MICs for $S$. aureus, even those well within the current susceptible range (MIC $2 \mu \mathrm{g} \mathrm{ml}^{-1}$ ) (CLSI, 2006a), might be a significant risk factor for vancomycin treatment failure (Tenover, 2008).

There is a paucity of data available from the Indian subcontinent surveying the occurrence of $S$. aureus isolates with reduced susceptibility to vancomycin. In this context, we performed a retrospective study to assess the trends in MIC levels for vancomycin susceptibility of $S$. aureus strains during a 5 year period in a tertiary hospital of India. Furthermore, a multiplex PCR for simultaneous detection of vanA and $v a n \mathrm{~B}$ genes, and the staphylococcal meticillin-resistance marker mecA, was performed on all the strains evaluated in this study.

MICs for vancomycin were determined by broth microdilution, as recommended by the Clinical and Laboratory Standard Institute (CLSI, 2006b), using panels prepared in-house. S. aureus isolates with vancomycin MICs of $\leqslant 2 \mu \mathrm{g} \mathrm{ml}^{-1}$ were considered as susceptible. VISA was considered as MICs of 4-8 $\mu \mathrm{g} \mathrm{ml}^{-1}$, and VRSA as MICs of $\geqslant 16 \mu \mathrm{g} \mathrm{ml}^{-1}$. Quality control was performed by using CLSI recommended reference strains. To detect genes coding for vancomycin and meticillin resistance, multiplex PCR assays were performed as described by RamosTrujillo et al. (2003).

A total of 632 S. aureus isolates was included in the analysis. The distribution of vancomycin MICs is shown in Table 1 according to the year of isolation. Overall, vancomycin MICs ranged from $0.5-2 \mathrm{mg} \mathrm{l}^{-1}$ $\left(\mathrm{MIC}_{50} 1 \mathrm{mg} \mathrm{l}^{-1}\right)$ for both meticillinsusceptible S. aureus (MSSA) and MRSA strains. Of note, the number of $S$. aureus isolates that had a vancomycin MIC of $\leqslant 0.5 \mu \mathrm{g} \mathrm{ml}^{-1}$ was extremely low $(1.7 \%)$. A gradual shift in vancomycin MICs from 1 to $2 \mu \mathrm{g} \mathrm{ml}^{-1}$ was observed among MRSA isolates during the 5 year period. The percentage of MRSA isolates with a vancomycin MIC of $1 \mu \mathrm{g} \mathrm{ml}^{-1}$ gradually decreased from $51.5 \%$ during 2004 to $37.7 \%$ in 2008 . This trend was statistically significant $(P=0.01)$. Interestingly, the percentage of MRSA isolates with a vancomycin MIC of $2 \mu \mathrm{g} \mathrm{ml}^{-1}$ gradually increased from $40.6 \%$ in 2004 to $61.3 \%$ in 2008 showing a significant trend of increasing MIC $(P<0.01)$. Similar trends were not noticeable in MSSA strains. No VRSA/VISA isolates were detected both by broth microdilution and multiplex PCR assay.

In this study, we report the shifting trends of vancomycin susceptibility patterns in a large tertiary care hospital in India (All India Institute of Medical Sciences). Although all S. aureus strains remained susceptible to vancomycin when breakpoints were applied, the increasing vancomycin MICs may adversely affect vancomycin activity against $S$. aureus strains. As observed by Sakoulas et al. (2004) the efficacy of vancomycin in the treatment of $S$. aureus infections decreased for isolates with vancomycin MICs of $1 \mu \mathrm{g}$ $\mathrm{ml}^{-1}$. The number of clinical failures for patients treated with vancomycin may rise as vancomycin MICs increase to $2 \mu \mathrm{g} \mathrm{ml}^{-1}$ (Sakoulas et al., 2004). It is not known whether the increasing number of $S$. aureus isolates with vancomycin MICs of $2 \mu \mathrm{g}$ $\mathrm{ml}^{-1}$ and the observed shift in vancomycin MICs from 1 to $2 \mu \mathrm{g} \mathrm{ml}^{-1}$ over the 5 years of our study represent a real clinical problem in the treatment of $S$. aureus infections with vancomycin.

The possible emergence and dissemination of VRSA strains is a serious health threat, and makes it absolutely necessary to optimize prevention strategies and fast detection methods. The multiplex PCR assay performed in this study is a rapid diagnostic method that can be used to facilitate the detection and identification of S. aureus or MRSA strains carrying vanA or $\operatorname{vanB}$. No strain with high level of vancomycin resistance due to vanA or van $\mathrm{B}$ was observed in this study. However, the emergence of vancomycin-resistant enterococci in our hospital (All India Institute of Medical Sciences) in recent years (Mathur et al., 2003; Dhawan et al., 2009) is a cause for concern, as vanA-type resistant genes can be transferred from enterococci to $S$. aureus thereby further exacerbating the potential for the emergence of VRSA in our hospital setting.

We have previously reported the genetic relatedness analysis of our MRSA strains by PFGE (Gadepalli et al., 2009). The MRSA strains with vancomycin MICs of $2 \mu \mathrm{g} \mathrm{ml}^{-1}$ belonged to PFGE clone III, which is the predominant multidrug resistant clone circulating in our hospital and shows genetic relatedness to the epidemic strain UK EMRSA-1. The propensity of clone III for resistance to different antimicrobials underscores the importance of the implementation of strategies to prevent further clonal spread of MRSA strains. 
Table 1. Number of S. aureus isolates tested for vancomycin susceptibility from 2004 to 2008 and vancomycin MICs

\begin{tabular}{|c|c|c|c|c|c|c|c|c|c|c|c|c|}
\hline \multirow[t]{3}{*}{ Year } & \multicolumn{3}{|c|}{ Total no. of isolates } & \multicolumn{9}{|c|}{ No. (percentage) of isolates with vancomycin MIC of: } \\
\hline & \multirow[t]{2}{*}{ MSSA } & \multirow[t]{2}{*}{ MRSA } & \multirow[t]{2}{*}{ Total } & \multicolumn{3}{|c|}{$\leqslant 0.5 \mu \mathrm{g} \mathrm{ml}^{-1}$} & \multicolumn{3}{|c|}{$1 \mu \mathrm{g} \mathrm{ml}^{-1}$} & \multicolumn{3}{|c|}{$2.0 \mu \mathrm{g} \mathrm{ml}^{-1}$} \\
\hline & & & & MSSA & MRSA & Total & MSSA & MRSA & Total & MSSA & MRSA & Total \\
\hline 2004 & 49 & 64 & 113 & $1(2.0)$ & $5(7.8)$ & $6(5.3)$ & $33(67.3)$ & $33(51.5)$ & $66(58.4)$ & $15(30.6)$ & $26(40.6)$ & $41(36.3)$ \\
\hline 2005 & 43 & 60 & 103 & $0(0.0)$ & $1(1.7)$ & $1(1.0)$ & $28(65.1)$ & $31(51.7)$ & $59(57.3)$ & $15(34.9)$ & $28(46.7)$ & $43(41.7)$ \\
\hline 2006 & 31 & 62 & 93 & $0(0.0)$ & $1(1.6)$ & $1(1.0)$ & $19(61.2)$ & $28(45.2)$ & $47(50.5)$ & $12(38.7)$ & $33(53.2)$ & $45(48.4)$ \\
\hline 2007 & 68 & 67 & 135 & $1(1.5)$ & $1(1.5)$ & $2(1.5)$ & $52(76.5)$ & $26(38.8)$ & $78(57.8)$ & $15(22.1)$ & $40(59.7)$ & $55(40.7)$ \\
\hline 2008 & 82 & 106 & 188 & $0(0.0)$ & $1(0.9)$ & $1(0.5)$ & $57(69.5)$ & $40(37.7)$ & 97 (51.6) & $25(30.5)$ & $65(61.3)$ & $90(47.9)$ \\
\hline Total & 273 & 359 & 632 & $2(0.7)$ & $9(2.5)$ & $11(1.7)$ & $189(69.2)$ & $158(44.0)$ & $347(54.9)$ & $82(30.0)$ & $192(53.5)$ & $274(43.4)$ \\
\hline$P$ value & & & & & & & 0.46 & 0.01 & 0.33 & 0.58 & $<0.01$ & 0.20 \\
\hline
\end{tabular}

$P$, Probability value based on $\chi^{2}$ test for a linear trend.

In conclusion, analysis of the evolutionary trend among S. aureus clinical isolates demonstrated a tendency toward increasing vancomycin MICs. Despite the absence of VRSA and VISA in our hospital, this increase in vancomycin MICs may raise more concerns about the potential failure of treatment of $S$. aureus infections with vancomycin. Such an MIC increase warrants continuous surveillance to allow timely detection and identification of the emergence of VRSA and VISA strains in Indian hospitals, since the increasing vancomycin MIC may indicate a first step towards VISA.

\section{Benu Dhawan, ${ }^{1}$ Ravisekhar Gadepalli, ${ }^{1}$ Chandrabhan Rao, ${ }^{1}$ Arti Kapil ${ }^{1}$ and V. Sreenivas ${ }^{2}$}

\section{${ }^{1}$ Department of Microbiology, All India Institute of Medical Sciences, New Delhi 29, India \\ ${ }^{2}$ Department of Biostatistics, All India Institute of Medical Sciences, New Delhi 29, India}

Correspondence: Benu Dhawan
(b_neha2002@yahoo.co.in)

Biedenbach, D. J., Moet, G. J. \& Jones, R. N. (2004). Occurrence and antimicrobial resistance pattern comparisons among bloodstream infection isolates from the SENTRY
Antimicrobial Surveillance Program (19972002). Diagn Microbiol Infect Dis 50, 59-69.

Chapman, A. L., Greig, J. M. \& Innes, J. A. (2005). MRSA in the community. N Engl J Med 353, 530-532.

CLSI (2006a). Performance Standards for Antimicrobial Susceptibility Testing, 16th informational supplement, M100-S16. Wayne, PA: Clinical and Laboratory Standards Institute.

CLSI (2006b). Methods for Dilution Antimicrobial Susceptibility Tests for Bacteria that Grow Aerobically, 7th edn, approved standard M7-A7. Wayne, PA: Clinical and Laboratory Standards Institute.

Dhawan, B., Gadepalli, R. \& Kapil, A. (2009). In vitro activity of daptomycin against Staphylococcus aureus and vancomycin-resistant Enterococcus faecium isolates associated with skin and soft tissue infections: first results from India. Diagn Microbiol Infect Dis 65, 196-198.

Fridkin, S. K., Hageman, J., McDougal, L. K., Mohammed, J., Jarvis, W. R., Perl, T. M. \&

Tenover, F. C, for the VancomycinIntermediate Staphylococcus aureus Epidemiology Study Group (2003).

Epidemiological and microbiological characterization of infections caused by Staphylococcus aureus with reduced susceptibility to vancomycin, United States, 1997-2001. Clin Infect Dis 36, 429-439.

Gadepalli, R., Dhawan, B., Kapil, A., Sreenivas, V., Jais, M., Gaind, R., Chaudhry, R., Samantaray, J. C. \& Udo, E. E. (2009). Clinical and molecular characteristics of nosocomial meticillin-resistant Staphylococcus aureus skin and soft tissue isolates from three Indian hospitals. J Hosp Infect 73, 253-263.
Hiramatsu, K., Aritaka, N., Hanaki, H., Kawasaki, S., Hosoda, Y., Hori, S., Fukuchi, Y. \& Kobayashi, I. (1997). Dissemination in Japanese hospitals of strains of Staphylococcus aureus heterogeneously resistant to vancomycin. Lancet 350, 1670-1673.

Mathur, P., Kapil, A., Chandra, R., Sharma, P. \& Das, B. (2003). Antimicrobial resistance in Enterococcus faecalis at a tertiary care centre of northern India. Indian J Med Res 118, 25-28.

NNISS (2004). National Nosocomial Infections Surveillance (NNIS) System report, data summary from January 1992 through June 2004, issued October 2004. Am J Infect Control 32, 470-485.

Ramos-Trujillo, E., Pérez-Roth, E., MéndezAlvarez, S. \& Claverie-Martín, F. (2003).

Multiplex PCR for simultaneous detection of enterococcal genes $v a n \mathrm{~A}$ and $\operatorname{van} \mathrm{B}$ and staphylococcal genes mecA, ileS-2 and femB. Int Microbiol 6, 113-115.

Robert, J., Bismuth, R. \& Jarlier, V. (2006). Decreased susceptibility to glycopeptides in methicillin-resistant Staphylococcus aureus: a 20 year study in a large French teaching hospital, 1983-2002. J Antimicrob Chemother 57, 506-510.

Sakoulas, G., Moise-Broder, P. A., Schentag, J., Forrest, A., Moellering, R. C., Jr \& Eliopoulos, G. M. (2004). Relationship of MIC and bactericidal activity to efficacy of vancomycin for treatment of methicillin-resistant Staphylococcus aureus bacteremia. J Clin Microbiol 42, 2398-2402.

Tenover, F. C. (2008). Vancomycin-resistant Staphylococcus aureus: a perfect but geographically limited storm? Clin Infect Dis $\mathbf{4 6}$, 675-677. 